\title{
Spectroscopic characterization and structural insights of 4- Coumarinyl-4-nitrobenzoate using vibrational and quantum chemical calculations
}

\author{
Kamuran SARAÇa,* \\ ${ }^{a}$ Bitlis Eren University, Faculty of Art and Sciences, Department of Chemistry, TR-13000, Bitlis, Turkey
}

\section{ARTICLE INFO}

\section{Article history:}

Received 31 March 2021

Received in revised form 19 April 2021

Accepted 27 April 2021

\section{Keywords:}

Density Functional Theory

MEP

HOMO-LUMO

\begin{abstract}
A BS TRACT
The aim of the study was to synthesize 4-Coumarinyl-4-nitrobenzoate and detect its experimental and theoretical properties. 4-Coumarinyl-4-nitrobenzoate was synthesized using by the nucleophilic adduction-separation reaction of aroyl compounds. In this context 4-coumarinyl 4-nitrobenzoate compound has been characterized both experimentally and theoretically by using quantum chemical calculations and spectral techniques. Quantum chemical calculations such as the molecular geometry, geometric structure, and geometric parameters of the title compound were calculated with the 6-311G (d, p) basis set. Quantum chemical calculations of electronic properties such as energy difference between HOMO-LUMO, chemical hardness and chemical softness were made. Molecular electrostatic potential (MEP) surface of 4-Coumarinyl-4-nitrobenzoate was obtained.
\end{abstract}

\section{Introduction}

Coumarins are oxygen-containing heterocyclic compounds isolated from plants, particularly green plants. It is known that there are about 1300 naturally derived coumarin derivatives. It occurs naturally in the fruits, bark, stems and leaves of many plants, including tonka beans, acacia, lavender, apricots, strawberries and cinnamon. In addition to natural coumarins isolated from plants, synthetic coumarin derivatives are also available. Coumarin and its derivatives are used in the treatment of different diseases and exhibit many biological properties such as anticoagulant, antiallergic, antibiotic, diuretic, anti-HIV, antibacterial. It is known that dicumaril and its derivatives, which are among the bisumarins, have anticoagulant effects and these compounds have a reducing effect on blood clotting and are used in the production of therapeutic drugs. Warfarin, which has 4-hydroxycoumarin structure, is the best known anticoagulant. Novabiosin and Chlorobiocin are coumarin derivatives with antibiotic and antibacterial properties and have poor farmo kinetic and toxic preventive properties [1]. Benzocumarins and benzochromones are compounds that have been studied recently due to their chiocological properties. These compounds are also used in the textile industry as a shine enhancer of synthetic fibers [2]. The photophysical properties of coumarin derivatives differ according to the groups connected in the structure and the solvent medium. Coumarin derivatives find a wide field of study as they have excellent responsiveness for important laser dyestuffs, non-linear optical chromophore and solubility dynamics studies in homogeneous solutions [3]. A significant number of coumarin dyes are used as blue, green and red additives in organic light emitting diodes (OLEDs). However, coumarin dyes easily selfabsorb at high concentrations due to intermolecular interactions. Therefore, it should always be used in appropriate concentrations to manufacture OLEDs with luminescent efficiency in light emitting materials [4]. Because of the use of coumarin derivatives in such a wide and diverse field, 4-Coumarinyl-4-nitro benzoate compound was synthesized for the first time in this study with the aim of using it in at least one of these fields. On the other hand, the 4-

* Corresponding author. Tel.: +90 4342222511

E-mail address: ksarac@beu.edu.tr 
Coumarinyl-4-nitrobenzoate compound has been characterized both experimentally and theoretically by using quantum chemical calculations and different spectral techniques. The data obtained from the theoretical calculations were used to calculate the global reactive descriptor and thermodynamic parameters.

\section{Materials and Methods}

\subsection{Experimental}

\section{Synthesis and physical properties}

To 4-hydroxycoumarin (1.62 g, $10 \mathrm{mmol}$ ) in dry pyridine ( 2 $\mathrm{mL}, 25 \mathrm{mmol}$ ) containing piperidine (1drop) at $0^{\circ}$ was slowly added 1.5 equivalent of 4 -nitro benzoyl chloride. The reaction mixture was poured into ice and dilute hydrochloric acid. The precipitate was filtered and washed with water. After shaking the product with $5 \%$ sodium bicarbonate solution, it was again filtered, washed and recrystallized with ethanol.<smiles>O=c1cc(O)c2ccccc2o1</smiles>

(I)<smiles>O=C(Oc1cc(=O)oc2ccccc12)c1ccc([N+](=O)[O-])cc1</smiles>

(II)
Figure 1. The reaction for the synthesis of title compound [5].

\subsection{Computational methods}

Gas-phase geometrical optimizations of title compound was performed by using the ab initio method. The relative energy for the two tautomers has been calculated by DFT method at the B3LYP/6-311G(d,p) level of theory [6].
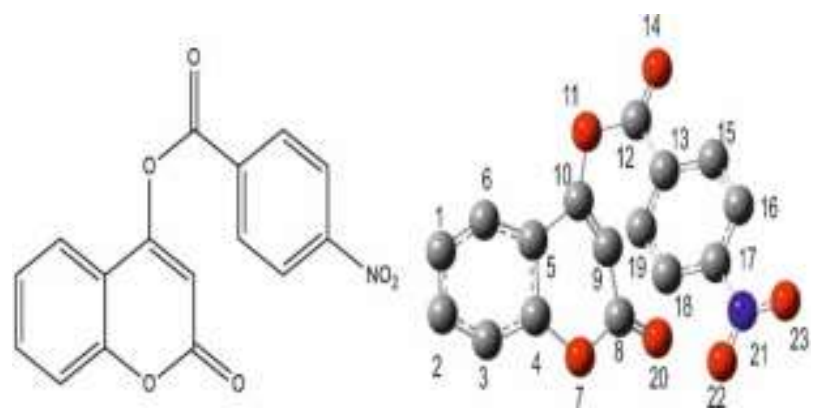

Figure 2. (a) Molecular structure of 4-Coumarinyl-4-nitrobenzoate (b) The theoretical geometric structure of the title compound (with B3LYP/6-311G(d,p) level).

\section{Results and Discussion}

\subsection{Atomics charge distributions in gas-phase and in solutionphase}

Atomic charge distributions in gas and solution phases The electronic charge distribution in a molecule as well as the nature of the molecular orbitals (i.e. antibonding, bonding or nonbonding) for certain atom pairs can be characterized using the technique of Mulliken charge distribution [7]. The formation of pairs of donors and acceptors including the charge transfer in the molecule is indicated by the charge distribution over the atoms. Table 1. shows the Mulliken atomic charges for carbon, nitrogen and oxygen atoms of 4Coumarinyl-4-nitrobenzoate determined at the B3LYP/6$311 G(d, p)$ level with the molecule in gas phase. Additionally, Table 1. also shows the obtained values of the three solvents (chloroform, water and ethanol) which were chosen to determine the solvent effect on the atomic charge distributions of 4-Coumarinyl-4-nitrobenzoate on the basis of the B3LYP/6$311 \mathrm{G}(\mathrm{d}, \mathrm{p})$ model. According to the Mulliken technique, the negative atomic charges of the 020 atom of pyrone ring, 022 and 023 atoms of benzoate are larger compared to the gas phase. On the other hand, the 020, 022 and 023 atoms have not show any significant change in atomic charge values between gas phase and solution phases.

\subsection{Nuclear magnetic resonance (NMR) spectra and Vibrational assignments}

The Hydrogen in the $1 \mathrm{H}$ NMR spectrum of the coumarin was observed as singlet at 7,10 ppm. The protons $(8 \mathrm{H})$ coumarin and benzoyl were observed as a multiplet in the range of 7.50$8.20 \mathrm{ppm}$. Chemical shift of all carbons in coumarin and benzoyl ring were shown between 115 and 138 ppm. The C-O stretching vibration in Coumarin was observed in the 1250$850 \mathrm{~cm}-1$ region [8]. In the present study, the $\mathrm{C}-\mathrm{O}$ streching vibration was observed at $1172 \mathrm{~cm}-1$. The $\mathrm{C}=0$ stretching frequency was observed in the range of $1600-1850 \mathrm{~cm}-1$ owing to its large change in dipolemoment and its characteristic frequency used to study a wide range of the compounds [9]. The $\mathrm{C}=0$ stretching vibration in title compound was observed at $1733 \mathrm{~cm}-1$. All these data confirm the structure of the compound. 
Table.1. Atomic charges (e) of the title compound in gas phase and solution phase.

\begin{tabular}{lllll}
\hline Atom & Gas & Chloroform & Ethanol & Water \\
\hline C1 & -0.334171 & -0.334170 & -0.334170 & 0.334170 \\
C2 & -0.252517 & -0.252518 & -0.252518 & -0.252518 \\
C3 & -0.406281 & -0.406281 & -0.406281 & -0.406281 \\
C4 & 0.545140 & 0.545140 & 0.545140 & 0.545140 \\
C5 & 0.128568 & -0.328569 & 0.128569 \\
C6 & -0.321010 & -0.534787 & -0.321010 \\
07 & -0.534787 & 1.157896 & -0.321010 & -0.534787 \\
C8 & 1.157896 & -0.649201 & -0.534787 & 1.157896 \\
C9 & -0.649202 & 0.536221 & -0.649201 \\
C10 & 0.536222 & -0.488570 & -0.67896101 & \\
011 & -0.488570 & 0.921118 & 0.536221 & -0.536221 \\
C12 & 0.921118 & -0.488570 & 0.488570 \\
C13 & 0.061170 & 0.921118 & 0.921118 \\
014 & -0.826672 & -0.826672 & 0.061170 & -0.826672 \\
C15 & -0.271796 & -0.271796 & -0.826672 & -0.271796 \\
C16 & -0.258750 & -0.258749 & -0.271796 & -0.258749 \\
C17 & 0.496510 & 0.496510 & -0.258749 & 0.496510 \\
C18 & -0.306465 & -0.306464 & 0.496510 & -0.306464 \\
C19 & -0.363628 & -0.363629 & -0.306464 & -0.363629 \\
020 & -0.826024 & -0.826025 & -0.363629 & -0.826025 \\
N21 & 0.811344 & 0.811344 & -0.826025 & 0.811344 \\
022 & -0.617032 & -0.617031 & 0.811344 & -0.617031 \\
023 & -0.618195 & -0.618195 & -0.617031 & -0.618195 \\
\hline
\end{tabular}

\subsection{The frontier molecular orbitals}

Frontier Molecular orbitals (FMO's) generally play an essential role in chemical reactions, UV-vis spectra, optical and electrical properties. Also recently, the energy gap $(\Delta \mathrm{E})$ of HOMO-LUMO has been used to prove the chemical activity and the bioactivity from intra molecular charge transfer [10]. The 3D plots of the HOMO-1,HOMO,LUMO,LUMO+1 orbitals were calculated at the B3LYP/6-311G (d,p) and shown in Figure 3. As seen from the Figure 3, the LUMO is located on nitro group and benzene ring, the HOMO is more located on the coumarin ring and partially over the carbonyl group.

The ability of electron accepting and giving defines for LUMO and HOMO, respectively. Besides, the HOMO-LUMO energies and their energy gap $(\Delta \mathrm{E})$ are calculated with the B3LYP/6$311 \mathrm{G}(\mathrm{d}, \mathrm{p})$ method. The global chemical reactivity and density functional descriptors of the title compound such as dipole moment $(\mu)$, electron affinity $(A)$, electronegativity $(\chi)$, chemical potential $(\mu \mathrm{o})$, ionization potential (I), global hardness $(\eta)$, electrophilicity index $(\omega)$ and global softness $(\zeta)$ are calculated from the energies of the HOMO and the LUMO at B3LYP/6-311G (d,p) basis set and are given in Table 2.

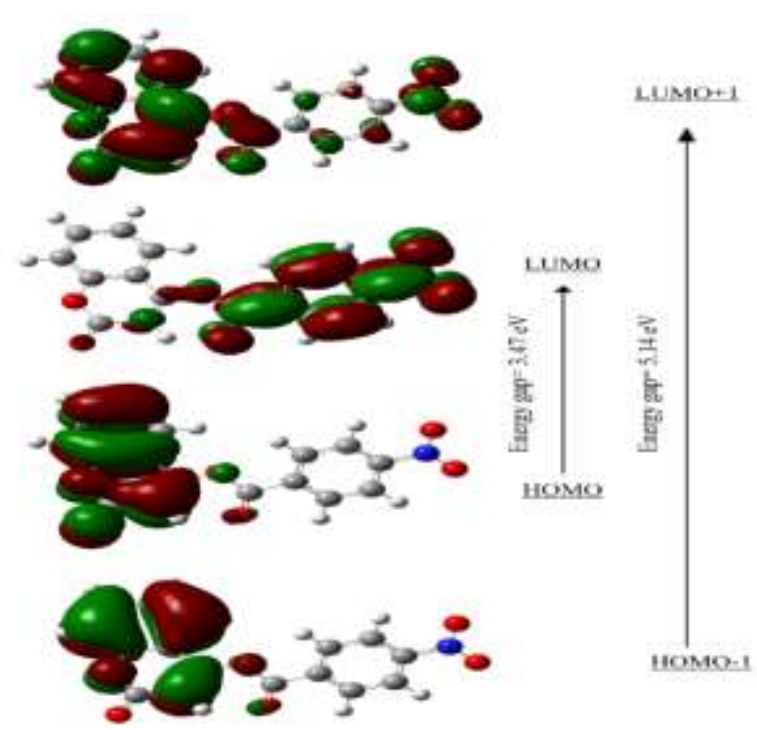

Figure 3. Molecular orbital surfaces and energy levels given in parentheses for the HOMO, HOMO-1, LUMO and LUMO-1 of the title compound computed at B3LYP/6-311G(d,p) level. 
Table 2. Electronic structure parameters of the title compound.

\begin{tabular}{ll}
\hline Parameters & $\underline{\text { B3LYP }}$ \\
\hline E-31G(d,p) \\
\hline Eномо $(\mathrm{eV})$ & -3.2264 \\
$\Delta \mathrm{E}=\mathrm{E}_{\text {LUMo- }}-\mathrm{E}_{\text {Hомо }}(\mathrm{eV})$ & -6.6969 \\
$\mathrm{~A}(\mathrm{eV})$ & 3.4705 \\
$\mathrm{I}(\mathrm{eV})$ & 3.2264 \\
$\zeta(\mathrm{eV})$ & 6.6969 \\
$\mu_{\mathrm{o}}(\mathrm{eV})$ & 0.5763 \\
$\eta(\mathrm{eV})$ & -4.9616 \\
$\omega(\mathrm{eV})$ & 1.7352 \\
$\chi(\mathrm{eV})$ & 7.0935 \\
\hline
\end{tabular}

These parameters can be calculated as follows [11].

When the chemical hardness is considered, a molecule having a small HOMO-LUMO energy gap means a soft molecule and large energy gap means a hard molecule. It may also relate to the stability of the molecule against hardness. That is, the molecule with least energy gap is less stable and more reactive. The calculated values of $\zeta$, 目, EHOMO ELUMO, $\Delta \mathrm{E}, \mu$ o and for the title compound are $0.57,1.73,6.69,3.22,3.47,-4.96$ and $4.96 \mathrm{eV}$, respectively. Taking into account the value of the energy between HOMO and LUMO, it is shown that charge transfer is within the title compound. Besides, as shown in Table 2, the chemical potential is negative and it means that the title compound is stable.

\subsection{Molecular electrostatic potential (MEP)}

Molecular electrostatic potential (MEP) is related to the electron density and serves a very useful descriptor to explain nucleophilic and electrophilic reaction regions, hydrogen bonding interactions, structure-activity relationship of molecules containing drugs and biomolecules [12]. Molecular electrostatic potential surfaces were mapped by using B3LYP/6$311 \mathrm{G}(\mathrm{d}, \mathrm{p})$ basis set to predict reactive sites of electrophilic and nucleophilic attacks for the title compound as shown in Figure 4.

The different values of the electrostatic potential are shown by different colors at the surface; namely, blue, green and red colors show the regions of the most positive electrostatic potential, zero potential, the regions of the most negative electrostatic potential, respectively [13].

Potential decreases in the order blue $>$ green $>$ yellow $>$ orange $>$ red. The positive regions related to nucleophilic reactivity (blue color), whereas the negative regions of MEP related to electrophilic reactivity (red and yellow colors). As seen in Figure 4, From the molecular, it is evident that the negative regions are mainly localize over the carbonyl group rather than oxygen atoms, the positive regions are mostly localized over hydrogen atoms.
Consequently, it is expected that the more electronegativity in the carbonyl group makes it the most reactive sites for electrophilic attack, whereas the hydrogen atoms are the most reactive for nucleophilic attack in title compound.

\section{Conclusions}

Electronic investigations of 4-Coumarinyl-4-nitro benzoate has been carried out were calculated using the B3LYP method using 6-311G (d,p) basis set show the molecular geometry parameters. By mapping an electrondensity isosurface with molecular electrostatic potential surface, information about the shape, size, and site of high electronegativity and charge distribution of the title compound has been obtained. Using the B3LYP/6-311G(d,p) method, which provide, the energies of HOMO\&LUMO and their orbital energy gaps were calculated as well as the physical and structural properties of the molecules. $3.47 \mathrm{eV}$ was found to be the frontier orbital energy gap (EHOMO-ELUMO). With the aid of the theoretical calculations at the B3LYP/6-311G(d,p) level. The positive potential sites are around the hydrogen atoms and the negative potential sites are on electronegative atoms, which are shown by the MEP map. The region from where the compound may have intermolecular interactions can be understood based on this information provided.

\section{References}

[1] Bouasla, S., Amaro-Gahete J., Esquivel, D., López, M.I., Jiménez-Sanchidrián, C., Teguiche, M., Romero-Salguero, F. 2017. Coumarin derivatives solvent-free synthesis under microwave irradiation over heterogeneous solid catalysts. Molecules, 22 (12), 2072-2080.

[2] Kolancılar, H. 2019. DFT Yöntemi Kullanılarak 1,3-Bis-\{(2Aminobenzoil) Amino\} Propanın Teorik Hesaplamaları ve Bu Değerlerin Literatürdeki Deneysel Değerler ile Karşılaștırılması. Düzce Üniversitesi Bilim ve Teknoloji Dergisi, 7 (3), 1319-1334.

[3] Abdel, M.S.A., Hamed E., Saif, M., Hafez S. 2018. Binding, and thermodynamics of $\beta$ cyclodextrin inclusion complexes with some coumarin laser dyes and coumarin-based enzyme substrates: a simulation study. Journal of Inclusion Phenomena and Macrocyclic Chemistry, 92 (3), 319-327.

[4] Yu, T., Zhu, Z., Bao Y., Zhao, Y., Liu, X., Zhang, H. 2017. Investigation of novel carbazolefunctionalized coumarin derivatives as organic luminescent materials. Dyes and Pigments, 147 (1), 260-269.

[5] Babinski, D., Soltani, O., Frantz, D.E. 2008. Stereoselective synthesis of acetoacetate-derived enol triflates. Organic Letters, 10 (13), 2901-2904.

[6] Vignale, G., Rasolt, M., 1987. Density-functional theory in strong magnetic fields. Phys. Rev. Lett. 59, 2360-2363.

[7] Arunagiri, C., Arivazhagan, M., Subashini, A., Maruthaiveeran, N. 2014. Theoretical and experimental calculations, Mulliken charges and thermodynamic properties of 4-chloro-2nitroanisole. Spectrochim. Acta Part A Mol.Biomol. Spectrosc, 131, 647-656. 
[8] Karakas, S,E., Dereli, Ö. 2013. Molecular structure and vibrational spectra of 7-Methoxy-4-methylcoumarin by density functional method. J Mol Struct.1052, 214-20.

[9] Lin-Vien, D., Fateley, WG., Grasselli, J.G., Colthup, N.B.,1991. The Handbook of Infrared and Raman Characteristic Frequencies of Organic Molecules, Academic Press.

[10] Fleming, J., 1976. Frontier Orbitals and Organic Chemical Reactions, John Wiley, London.

[11] Lee, C., Yang, W., Parr, R.G., 1988. Local softness and chemical reactivity in the molecules $\mathrm{CO}, \mathrm{SCN}-$ and H2CO. Journal of Molecular Structure: Theochem, 163, 305-313.
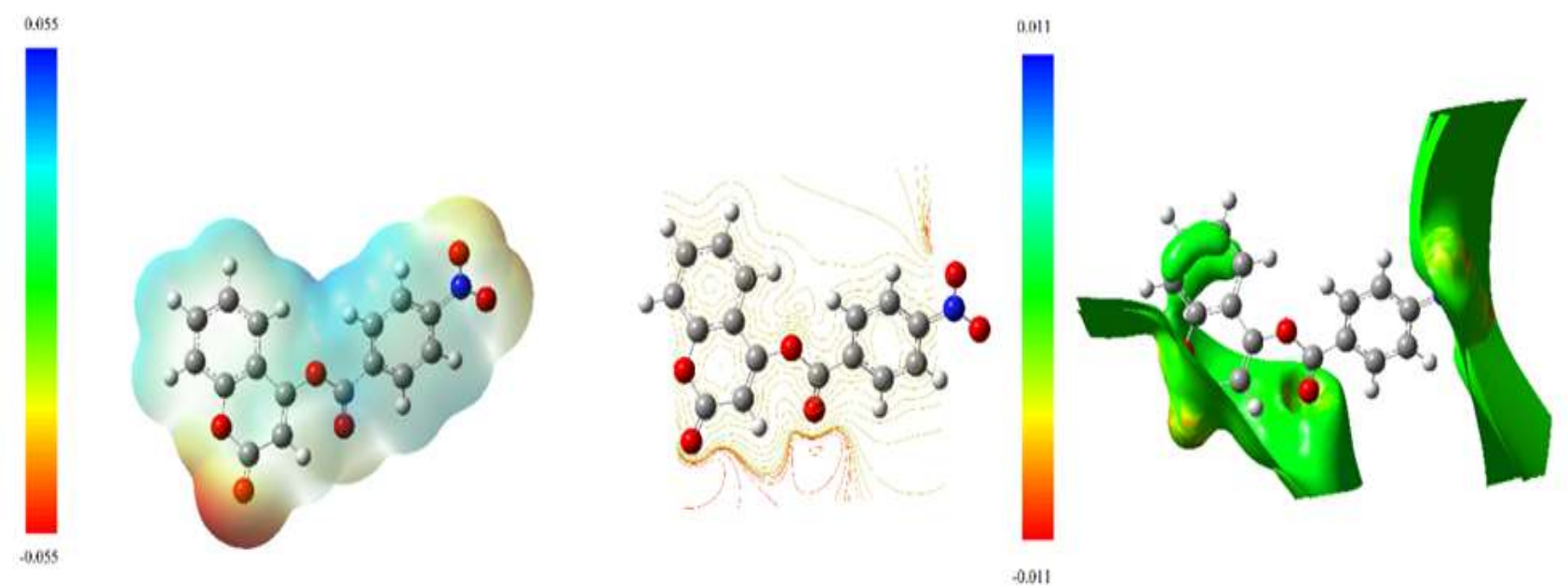

[12] Luque, F.J., López, J.M., Orozco, M., 2000. Perspective on Electrostatic interactions of a solute with a continuum. A direct utilization of ab initio molecular potentials for the prevision of solvent effects. Theoretical Chemistry Accounts, 103, 343-345.

[13] Sebastian. S., Sundaraganesan. N., 2010. The UV) and NBO analysis of 4-Hydroxypiperidine by density functional method. Spectrochimica Acta Part A: Molecular and Biomolecular Spectroscopy, 75 (3), 941 952. spectroscopic (FT-IR, FT-IR gas phase, FT-Raman and

Figure 4. Molecular electrostatic potential maps of the title compound 